\title{
Preclinical Evaluation of RYM1, a Matrix Metalloproteinase- Targeted Tracer for Imaging Aneurysm
}

\author{
Jakub Toczek*1,2, Yunpeng Ye*1,2, Kiran Gona ${ }^{1,2}$, Hye-Yeong Kim ${ }^{1,2}$, Jinah Han ${ }^{1,2}$, Mahmoud Razavian ${ }^{1,2}$, \\ Reza Golestani ${ }^{1,2}$, Jiasheng Zhang ${ }^{1,2}$, Terence L. Wu ${ }^{3}$, Jae-Joon Jung ${ }^{1,2}$, and Mehran M. Sadeghi ${ }^{1,2}$ \\ ${ }^{I}$ Cardiovascular Molecular Imaging Laboratory, Section of Cardiovascular Medicine and Yale Cardiovascular Research Center, Yale \\ University School of Medicine, New Haven, Connecticut; ${ }^{2}$ Veterans Affairs Connecticut Healthcare System, West Haven, Connecticut; \\ and ${ }^{3}$ Yale West Campus Analytical Core, Yale University, West Haven, Connecticut
}

\begin{abstract}
Matrix metalloproteinases (MMPs) play a key role in abdominal aortic aneurysm (AAA) development. Accordingly, MMP-targeted imaging provides important information regarding vessel wall biology in the course of aneurysm development. Given the small size of the vessel wall and its proximity with blood, molecular imaging of aneurysm optimally requires highly sensitive tracers with rapid blood clearance. To this end, we developed a novel hydrosoluble zwitterionic MMP inhibitor, RYM, on the basis of which a pan-MMP tracer, RYM1, was designed. Here, we describe the development and preclinical evaluation of RYM1 in comparison with RP805, a commonly used pan-MMP tracer in murine models of aneurysm. Methods: The macrocyclic hydroxamate-based pan-MMP inhibitor coupled with 6-hydrazinonicotinamide, RYM1, was synthesized and

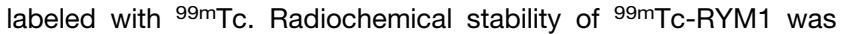
evaluated by radio-high-performance liquid chromatography analysis. Tracer blood kinetics and biodistribution were compared with 99mTc-RP805 in C57BL/6J mice $(n=10)$. ${ }^{99 m T c-R Y M 1 ~ b i n d i n g ~ t o ~}$ aneurysm and specificity were evaluated by quantitative autoradiography in apolipoprotein $\mathrm{E}$-deficient $\left(\mathrm{apoE}^{-/-}\right)$mice with $\mathrm{CaCl}_{2-}$ induced carotid aneurysm $(n=11)$. Angiotensin II-infused apoE ${ }^{-/-}$ $(n=16)$ mice were used for small-animal SPECT/CT imaging. Aortic tissue MMP activity and macrophage marker CD68 expression were assessed by zymography and reverse-transcription polymerase chain reaction. Results: RYM1 showed nanomolar range inhibition constants for several MMPs. ${ }^{99 m T c-R Y M 1}$ was radiochemically stable in mouse blood for $5 \mathrm{~h}$ and demonstrated rapid renal clearance and lower blood levels in vivo compared with ${ }^{99 \mathrm{~m} T c-}$ RP805. ${ }^{99 m T c-R Y M 1 ~ b i n d i n g ~ t o ~ a n e u r y s m ~ a n d ~ i t s ~ s p e c i f i c i t y ~ w e r e ~}$ shown by autoradiography in carotid aneurysm. Angiotensin II infusion in apoE ${ }^{-1-}$ mice for $4 \mathrm{wk}$ resulted in AAA formation in $36 \%$ (4/11) of surviving animals. In vivo ${ }^{99 m T c-R Y M 1 ~ s m a l l-a n i m a l ~ S P E C T / ~}$ CT images showed higher uptake of the tracer in AAA than nondilated aortae. Finally, aortic uptake of ${ }^{99 m T c-R Y M 1}$ in vivo correlated with aortic MMP activity and CD68 expression. Conclusion: The newly developed pan-MMP inhibitor-based tracer ${ }^{99 m T c-R Y M 1}$ displays favorable pharmacokinetics for early vascular imaging and enables specific detection of inflammation and MMP activity in aneurysm.
\end{abstract}

Received Dec. 28, 2016; revision accepted Mar. 20, 2017.

For correspondence or reprints contact: Mehran M. Sadeghi, Yale Cardiovascular Research Center, 300 George St., \#770F, New Haven, CT 06511.

E-mail: mehran.sadeghi@yale.edu

${ }^{*}$ Contributed equally to this work.

Published online Mar. 30, 2017.

COPYRIGHT (C 2017 by the Society of Nuclear Medicine and Molecular Imaging.
Key Words: abdominal aortic aneurysm; molecular imaging; matrix metalloproteinase inhibitor; SPECT/CT; inflammation

J Nucl Med 2017; 58:1318-1323

DOI: 10.2967/jnumed.116.188656

\section{A}

bdominal aortic aneurysm (AAA) accounts for 10,00015,000 recorded deaths, mainly due to rupture, in the United States each year. Current clinical guidelines for surgical repair of AAA are based on aneurysm size, expansion rate, and clinical symptoms (1). However, a significant portion of AAA ruptures occurs in patients who do not meet the criteria for AAA repair, and some large AAAs may remain stable for many years. As such, new risk-stratification tools are needed to overcome the limitations of the current approach to patient selection for AAA repair. Molecular imaging targeted at the determinants of AAA expansion and rupture appears particularly promising in this regard (2). Matrix metalloproteinase (MMP) activation is a main pathophysiologic feature of AAA and is believed to be closely related to aneurysm progression and risk of rupture $(3,4)$. Accordingly, molecular imaging of MMP activation may be a useful tool for AAA risk stratification.

We have demonstrated the feasibility of MMP-targeted imaging and its predictive value for AAA expansion and rupture in a mouse model of AAA (5). However, the relatively slow blood clearance of ${ }^{99 \mathrm{~m} T c-R P 805}$, the tracer used in these studies, which mandates delaying imaging to at least $2 \mathrm{~h}$ after injection, is a relative barrier to further development of this agent for clinical applications. Moreover, the limited water solubility of RP805 precursor has hampered blocking studies aimed at defining the specific component of tracer uptake in various tissues in vivo.

To address these limitations, we developed a novel macrocyclic hydroxamate pan-MMP inhibitor, RYM, with improved water solubility (6). Here, we show that the hydrazinonicotinamidecontaining RYM conjugate (RYM1) retains MMP binding potential. In comparison with ${ }^{99 \mathrm{~m} T c-R P 805,}{ }^{99 m} \mathrm{Tc}-\mathrm{RYM} 1$ has a faster blood clearance, which is favorable for early-time-point imaging. In vivo evaluation by autoradiography and smallanimal SPECT imaging in 2 murine models of aneurysm, namely calcium chloride $\left(\mathrm{CaCl}_{2}\right)$-induced carotid aneurysm and Ang IIinduced AAA, demonstrated specific uptake of 99mTc-RYM1 in aneurysm in correlation with vessel wall MMP activity and inflammation. 


\section{MATERIALS AND METHODS}

\section{Synthesis and Characterization of RYM1 and RP805}

RP805 precursor was synthesized as described (7). The macrocyclic hydroxamate-based pan-MMP inhibitor RYM and its 6-hydrazinonicotinamide conjugate RYM1 were synthesized starting from an antisuccinic acid derivative, (2R,3S)-3-(tert-butoxycarbonyl)-2-iso-butylhex-5-enoic acid, in a 15-step reaction via a macrocyclic hydroxamic acid as a key intermediate (Supplemental Fig. 1; supplemental materials are available at http://jnm.snmjournals.org). The identity and purity of RYM1 were confirmed by liquid chromatography mass spectroscopy, ${ }^{1} \mathrm{H} /{ }^{13} \mathrm{C}$ nuclear MR spectroscopy, and analytic high-performance liquid chromatography (HPLC).

\section{MMP Affinity Assays}

RYM1 inhibition constants $\left(\mathrm{K}_{\mathrm{i}}\right)$ for recombinant human MMP-2, $-7,-9,-12$, and -13 (R\&D Systems) were evaluated in vitro using a fluorogenic MMP activity assay based on Mca-KPLGL-Dpa-AR-NH2 fluorogenic peptide substrate (R\&D Systems), as previously described with minor modifications $(8)$.

\section{Radiochemistry and Stability Analysis}

RP805 was labeled with ${ }^{99 \mathrm{~m}} \mathrm{Tc}$, in the presence of 3,3',3"-phosphanetriyltris (benzenesulfonic acid) trisodium salt and tricine coligands in a Sn-free formulation, as previously described (7). RYM1 was similarly labeled with ${ }^{99 \mathrm{~m}} \mathrm{Tc}$ to give ${ }^{99 \mathrm{~m}} \mathrm{Tc}-\mathrm{RYM} 1 \mathrm{in}$ a high radiochemical yield. Radiolabeling quality control was performed by reversed-phase radioHPLC analysis (HPLC system 2489; Waters) with a flow rate of $1 \mathrm{~mL} /$ min using an analytic column (Jupiter, $4 \mu \mathrm{m}$ Proteo $90 \AA$ A Phenomenex) with gradients of solvent $\mathrm{A}(0.16 \%$ ammonium formate in aqueous solution) and solvent B $(0.16 \%$ ammonium formate in $90 \%$ acetonitrile). The HPLC gradients were programmed as follows: $10 \%$ B for 2 min, $10 \%-70 \%$ B in 5 min, $5 \min 70 \%$ B, and 70\%-90\% B in 5 min. In vitro and in vivo stability of the tracer were analyzed by radio-HPLC after in vitro incubation in mouse blood at $37^{\circ} \mathrm{C}$ for up to $5 \mathrm{~h}$ and from urine collected at $2 \mathrm{~h}$ after tracer injection, respectively.

\section{Animals}

C57BL/6J mice $(n=10)$ were used to study tracer biodistribution and stability. Ten-week-old male apolipoprotein E-deficient $\left(\mathrm{apoE}^{-1-}\right)$ mice $(n=11)$ on a high-fat diet for a week were used for carotid aneurysm induction, as previously described (9). Briefly, under isoflurane anesthesia, the common carotid arteries were isolated and a solution of either $10 \% \mathrm{CaCl}_{2}$ or $0.9 \% \mathrm{NaCl}$ was applied periadventitially for $20 \mathrm{~min}$. In addition, 16-wk-old male apoE ${ }^{-1-}(n=16)$ mice were infused with human angiotensin II (Ang II, 1,000 ng/kg/min; Calbiochem), delivered by a subcutaneous osmotic minipump (model 2004; Alzet) implanted under anesthesia (isoflurane, 2\%) (10). All animals were originally purchased from Jackson Laboratories, and the experiments were performed according to the regulations of the Institutional Animal Care and Use Committees of Yale University and VA Connecticut Health Care System.

\section{Biodistribution}

For comparison of ${ }^{99 \mathrm{~m} T c-R Y M 1}$ and ${ }^{99 m} \mathrm{Tc}-\mathrm{RP} 805$ biodistribution, C57BL/6J mice were injected intravenously with $16 \pm 5 \mathrm{MBq}$ of either 99mTc-RYM1 $(n=5)$ or ${ }^{99 m}$ Tc-RP805 $(n=5)$. To investigate biodistribution in apoE $\mathrm{E}^{-/-}$mice, animals at $7 \mathrm{wk}$ after periadventitial application of $\mathrm{CaCl}_{2}$ or $\mathrm{NaCl}$ to carotid arteries were injected with $31 \pm 14$ $\mathrm{MBq}(14 \pm 2 \mu \mathrm{g} / \mathrm{kg})$ of ${ }^{99 \mathrm{mTc}} \mathrm{T}-\mathrm{RYM} 1$, with $(n=5)$ or without $(n=6)$ preinjection of an excess of the parent inhibitor, RYM $(23 \pm 8 \mathrm{mg} / \mathrm{kg})$. Animals were kept under anesthesia for $60 \mathrm{~min}$, and blood samples were collected at various times after injection. Tissue samples and body fluids were collected and weighed at $2 \mathrm{~h}$ after injection, and measured for their radioactivity by $\gamma$-well counting (WIZARD2; PerkinElmer). Data were expressed as percentage injected dose per gram of tissue or milliliter of blood.

\section{Quantitative Autoradiography}

In apo $\mathrm{E}^{-1-}$ mice at $7 \mathrm{wk}$ after periadventitial application of $\mathrm{CaCl}_{2}$ or $\mathrm{NaCl}$ to carotid arteries, the aorta and carotid arteries were dissected from surrounding adherent tissues under a stereoscopic microscope (MZ9.5; Leica) at $2 \mathrm{~h}$ after tracer injection. The tissues were placed on a phosphor screen (MultiSensitive Phosphor Screen; PerkinElmer) along with standards of known activity. The phosphor screen was scanned with a phosphor imager (Typhoon Trio; GE Healthcare Life Sciences), and the digitalized images were quantified by drawing regions of interest around various tissues to determine tissue activity (Fiji/ImageJ software; National Institutes of Health)

\section{In Vivo Small-Animal SPECT/CT Imaging}

After 4 wk of Ang II infusion, the surviving apoE ${ }^{-1-}$ mice received an intravenous injection of $40 \pm 7 \mathrm{MBq}$ of ${ }^{99 \mathrm{~m} T c-R Y M 1}$ and at $1 \mathrm{~h}$ after injection underwent small-animal SPECT/CT imaging, as previously described with minor modifications (5). Briefly, the imaging protocol involved 64 circular 30 -s projections using a dual-head smallanimal SPECT/CT system with 1-mm pinhole collimators (X-SPECT; $\gamma$-Medica). The animals were injected with $60 \mu \mathrm{L}$ of a CT contrast agent (ExiTron nano 12000; Miltenyi Biotec) after ${ }^{99 m}$ Tc-RYM1 administration. Contrast-enhanced CT $(50 \mathrm{kVp}, 512$ projections $)$ was performed immediately after SPECT acquisition. For image analysis, cylindric regions of interest $(2 \times 2 \times 3 \mathrm{~mm})$ were drawn around the area with the highest ${ }^{99 \mathrm{~m} T c-R Y M 1}$ signal along the suprarenal abdominal aorta, identified on the basis of CT images and anatomic landmarks. The size of the region of interest was adjusted to exclude uptake in surrounding tissues. To quantify residual blood activity, regions of interest were drawn over the left ventricle cavity.

\section{Tissue Analysis}

After small-animal SPECT/CT imaging, the suprarenal abdominal aorta was rapidly cleaned from adherent tissues under stereoscopic microscope and frozen in optimal-cutting-temperature compound. To determine the maximal external diameter, 5 - $\mu$ m-thick serial sections of the abdominal aorta (typically 10 sections, $200-300 \mu \mathrm{m}$ apart) were used for morphometric analysis (Fiji/ImageJ software; National Institutes of Health) after hematoxylin and eosin staining. Adjacent tissues were processed to extract protein and RNA at the sites of maximum uptake observed on SPECT images, identified on the basis of anatomic landmarks.

\section{Zymography}

Aortic tissue was lysed in a lysis buffer $(\mathrm{NaCl} 0.3 \mathrm{M}$, Tris $50 \mathrm{mM}$, Triton X-100 1\%, cOmplete Protease Inhibitor Cocktail [SigmaAldrich]), and protein concentration was measured using a colorimetric assay (Protein Assay Dye Reagent Concentrate [Bio-Rad], BioMate 3 [Thermo Scientific]). MMP activity was assessed in $1 \mu \mathrm{g}$ of protein lysate using a fluorometric zymography assay (SensoLyte 520 Generic MMP Activity Kit; AnaSpec), according to the manufacturer's instructions and presented in relative arbitrary units.

\section{Quantitative Reverse Transcription Polymerase Chain Reaction}

RNA was isolated from aortic tissue using a GenElute Mammalian Total RNA Miniprep Kit (Sigma-Aldrich) and reverse-transcribed using a QuantiTect Reverse Transcription Kit (Qiagen). Quantitative reverse transcription polymerase chain reaction analysis was performed with a 7500 Real-Time PCR System (Applied Biosystems) using the following primers and probe sets (MMP-2: Mm00439498_m1; MMP-9: Mm00442991_m1; MMP-12: Mm0050054_m1; CD68: Mm03047343_m1; $\beta$-actin: Mn00607939_s1, TaqMan Gene Expression Assays, Thermo Fisher Scientific), according to the manufacturer's instructions. CD68 and MMP gene expression were normalized to $\beta$-actin. 


\section{Statistical Analysis}

All data are presented as mean $\pm \mathrm{SD}$. The Mann-Whitney $U$ test was used to compare datasets from 2 experimental groups. Two-way ANOVA with post hoc Bonferroni adjustment was used to compare blood activity between groups. Spearman rank correlation was used to assess the significance of correlations (Prism 7; GraphPad). A $P$ value below 0.05 was considered statistically significant.

\section{RESULTS}

\section{In Vitro MMP Binding}

RYM1 displayed high binding affinity $\left(\mathrm{K}_{\mathrm{i}}\right)$ for all recombinant human MMPs evaluated (Table 1). Of these, the greatest affinity was observed for MMP-12 $\left(\mathrm{K}_{\mathrm{i}}, 2.2 \pm 0.5 \mathrm{nM}\right)$, whereas the affinity for MMP-7 was the lowest $\left(\mathrm{K}_{\mathrm{i}}, 33.2 \pm 5.0 \mathrm{nM}\right)$.

\section{Radiochemistry}

Radio-HPLC analysis of ${ }^{99 \mathrm{~m}}$ Tc-labeled RYM1 showed a single radiolabeled peak (Fig. 1A). The radioactive material extracted after in vitro incubation of ${ }^{99 \mathrm{~m} T c-R Y M 1}$ in mouse blood for up to $5 \mathrm{~h}$ (Supplemental Fig. 2) or urine collected from mice $2 \mathrm{~h}$ after intravenous injection of the tracer (Fig. 1B) showed similar radioHPLC profiles with a single major peak at a similar retention time without significant degradation. As expected, ${ }^{99 m} \mathrm{Tc}-\mathrm{RYM} 1$ showed high water solubility $\left(\log \mathrm{P}_{\mathrm{n} \text {-octanol/water }}=-4.0 \pm 0.1\right)$.

\section{Biodistribution and Clearance}

The biodistribution and blood clearance of ${ }^{99 \mathrm{~m} T c-R Y M 1}$ were studied in comparison with ${ }^{99 \mathrm{~m} T c-R P 805}$ in C57BL/6J mice. 99mTc-RYM1 had significantly lower blood-pool activity at $1 \mathrm{~h}$ after injection $(1.1 \pm 0.1$ vs. $2.5 \pm 1.2$ percentage injected dose per $\mathrm{mL}$, respectively, for ${ }^{99 \mathrm{~m} T c-R Y M 1}$ and ${ }^{99 \mathrm{~m} T c-R P 805, ~} P<$ 0.05 ) (Fig. 2A). This difference was primarily reflected in the initial activity values, suggesting a difference in first-pass clearance of the tracer from blood. Interestingly, despite a lower blood level, ${ }^{99 m}$ Tc-RYM1 tissue uptake at $2 \mathrm{~h}$ after injection was significantly higher than ${ }^{99 \mathrm{~m}} \mathrm{Tc}-\mathrm{RP} 805$ in several organs, but not in the normal aorta. Both tracers displayed a high activity in the kidneys and urine, indicative of renal clearance. However, contrary to the animals injected with ${ }^{99 \mathrm{~m}} \mathrm{Tc}-\mathrm{RYM} 1$ who had limited bile activity, animals injected with 99mTc-RP805 showed elevated activity in the bile (Fig. 2B).

\section{In Vivo Binding to Carotid Aneurysm and Specificity}

99mTc-RYM1 binding to carotid aneurysm and specificity in vivo were evaluated in apoE ${ }^{-1-}$ mice at $7 \mathrm{wk}$ after periadventitial application of $\mathrm{CaCl}_{2}$ to left carotid arteries. Similar to C57BL/6J mice, 99mTc-RYM1 (31 $\pm 14 \mathrm{MBq}$, intravenously) displayed a fast blood

TABLE 1

Inhibition Constant $\left(\mathrm{K}_{\mathrm{i}}\right)$ Values of RYM1 for Recombinant Human MMP-2, -7, -9, -12, and -13

\begin{tabular}{lr}
\hline Protease & \multicolumn{1}{c}{$\mathrm{K}_{\mathrm{i}}(\mathrm{nM})$} \\
\hline rhMMP-2 & $10.4 \pm 0.0$ \\
rhMMP-7 & $33.2 \pm 5.0$ \\
rhMMP-9 & $17.9 \pm 8.0$ \\
rhMMP-12 & $2.2 \pm 0.5$ \\
rhMMP-13 & $16.9 \pm 2.7$ \\
\hline rh $=$ recombinant human. \\
\hline
\end{tabular}

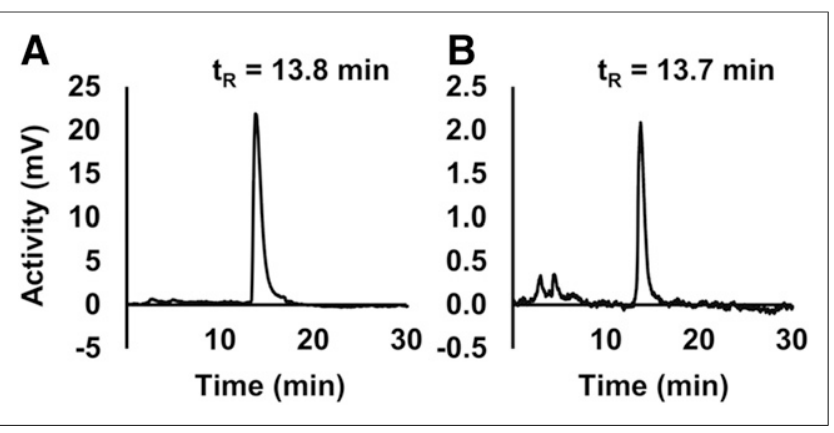

FIGURE 1. 99mTc-RYM1 stability. Representative radiochromatograms of ${ }^{99 \mathrm{~m} T c-R Y M 1}$ after radiolabeling $(A)$ and in urine collected from a C57BL/6J mouse at $2 \mathrm{~h}$ after injection $(\mathrm{B}) . \mathrm{t}_{\mathrm{R}}=$ retention time.

clearance, resulting in a residual blood-pool activity at 1 and $2 \mathrm{~h}$ after injection of $1.8 \pm 0.2$ and $1.3 \pm 0.1$ percentage injected dose per $\mathrm{mL}$, respectively. Autoradiographic evaluation of the carotids and aorta harvested at $2 \mathrm{~h}$ showed high uptake of the tracer in the left carotid artery aneurysm ( $n=6$, Fig. 3). Preinjection of an excess of the parent pan-MMP inhibitor RYM led to a 4.6-fold decrease in carotid aneurysm tracer uptake $(n=5, P<0.01)$. Albeit to a smaller degree, tracer uptake was also reduced in the aorta, resulting in a significant 1.8-fold decrease in the aneurysm-to-aorta uptake ratio by autoradiography under blocking conditions $(P<0.05$, Fig. 3). Importantly, preinjection of the parent pan-MMP inhibitor RYM significantly reduced ${ }^{99 \mathrm{~m}} \mathrm{Tc}-\mathrm{RYM} 1$ uptake, as assessed by $\gamma$-well counting in all tissues evaluated, but not in the bile (Supplemental Fig. 3), indicating a certain degree of systemic MMP activation in these mice.

\section{9mTc-RYM1 Imaging in AAA}

Ang II infusion resulted in the death of $31 \%(5 / 16)$ of apoE $^{-1-}$ mice within $4 \mathrm{wk}$. The surviving animals underwent ${ }^{99 \mathrm{~m} T c-R Y M 1}$ small-animal SPECT/CT imaging at $1 \mathrm{~h}$ after tracer injection. On visual and quantitative analysis of the images, a range of tracer uptake was detectable in suprarenal abdominal aortae of these animals. Accordingly, whereas in a subset of animals the aortic ${ }^{99 \mathrm{~m}} \mathrm{Tc}-$ RYM1 signal was readily detectable on in vivo SPECT/CT images, other animals displayed only modest uptake of the tracer in their suprarenal abdominal aortae (Fig. 4; Supplemental Fig. 4).

Visual examination of the aorta at the time of tissue harvesting immediately after small-animal SPECT/CT image acquisition detected varying degrees of aortic remodeling. On the basis of this visual analysis, $25 \%$ of animals (4/16) showed major focal dilation and were classified as those with suprarenal AAA. Conversely, $44 \%$ of animals (7/16) showed no or only modest remodeling and were categorized as the low remodeling group. This visual categorization was confirmed on morphometric analysis of tissue sections, which showed a significantly higher maximal external aortic diameter in the AAA, compared with the low remodeling group (1.74 \pm 0.35 vs. $0.99 \pm 0.08 \mathrm{~mm}, P<0.01$; Fig. 5; Supplemental Fig. 5).

When categorized on the basis of aortic size, there was a significant difference in suprarenal aortic ${ }^{99 \mathrm{~m}} \mathrm{Tc}-\mathrm{RYM}$ signal between the AAA and low remodeling groups $(0.66 \pm 0.16$ vs. $0.40 \pm 0.18$ counts per voxel [cpv]/MBq, $P<0.05$, Fig. 4). Similarly, fluorometric assessment of MMP activity showed significantly higher MMP activity in the AAA group than the low remodeling group (Fig. 5). Consistent with the MMP specificity of the tracer, a significant correlation existed between the aortic ${ }^{99 \mathrm{~m}}$ Tc-RYM1 signal in vivo and MMP activity detected by zymography ex vivo $\left(r^{2}=0.65, P<0.01\right.$, Fig. 4). There was no difference in the left-ventricle blood-pool activity 


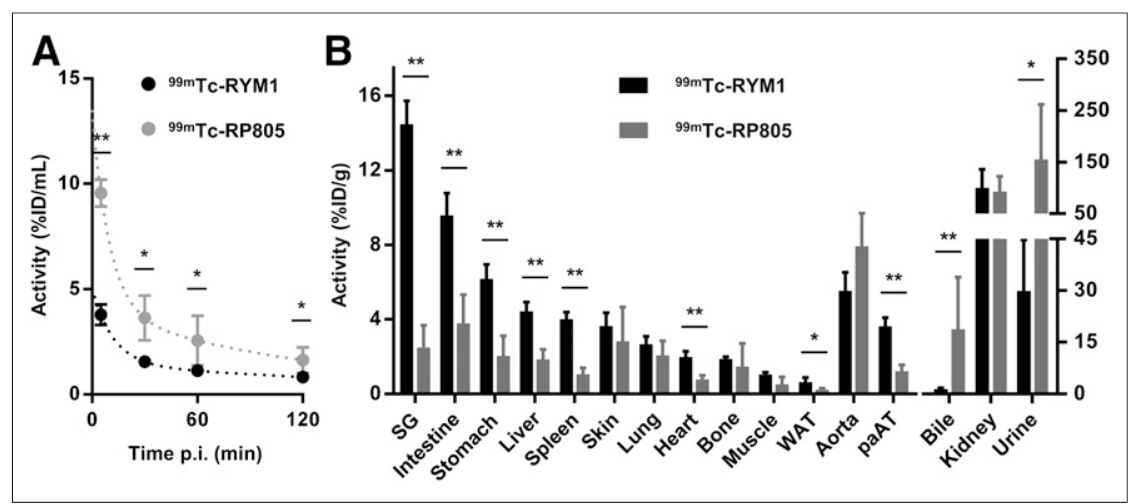

FIGURE 2. Tracer biodistribution and clearance. Blood kinetics $(A)$ and biodistribution $(B)$ at $2 \mathrm{~h}$ after $99 \mathrm{mTc}-\mathrm{RYM} 1$ and ${ }^{99 \mathrm{mTC}-R P 805}$ administration in C57BL/6J mice. ID = injected dose; pAT $=$ periaortic adipose tissue; p.i. $=$ after injection; $\mathrm{SG}=$ salivary glands; $\mathrm{WAT}=$ white adipose tissue. $n=5$ in each group. ${ }^{\star} P<0.05 .{ }^{\star \star} P<0.01$.

between the 2 groups of animals $(0.14 \pm 0.10$ vs. $0.12 \pm 0.08$ cpv/MBq, for AAA and low remodeling groups, respectively, $P=$ not significant).

\section{Gene Expression Analysis}

There was no significant difference in MMP-2 and MMP-9 gene expression between AAA and low remodeling groups, whereas macrophage marker CD68 and MMP-12 expression were significantly higher in the AAA than the low remodeling group (Supplemental Fig. 6). Moreover, aortic ${ }^{99 \mathrm{~m} T c-R Y M 1}$ signal in vivo correlated with CD68 and MMP-12 (Fig. 6), but not MMP2 and MMP-9 gene expression (Supplemental Fig. 7). Similarly, CD68 and MMP-12, but not MMP-2 and MMP-9, expression correlated with tissue MMP activity (Supplemental Table 1).

\section{DISCUSSION}

Here, we present the preclinical evaluation of a novel, pan-MMP inhibitor-based radiotracer, ${ }^{99 \mathrm{~m} T c-R Y M 1}$, rationally designed to address the shortcomings for clinical translation of ${ }^{99 \mathrm{~m} T c-R P 805,}$ a commonly used preclinical MMP-targeting SPECT tracer for cardiovascular applications. Our data demonstrate high affinity of this novel tracer for a set of MMPs involved in aneurysm development, its good radiochemical stability, favorable properties for vascular imaging, and specific uptake in aneurysm in vivo that correlates with tissue MMP activity and inflammation.

Increased MMP activation is a key feature of aneurysm and plays a central role in aortic remodeling. Accordingly, in vivo imaging of MMP activation may help predict the evolution of the disease and guide therapeutic decisions. The feasibility and potential value of in vivo MMP-targeted imaging in cardiovascular pathology has been shown in preclinical studies (11-18). Many of these studies have used a macrocyclic pan-MMP targeting tracer, ${ }^{99 \mathrm{~m}} \mathrm{Tc}-\mathrm{RP} 805$, which, based on existing preclinical data, is well-positioned to serve as a lead agent for further development. Despite the wealth of the available preclinical data, several shortcomings, including a relatively slow blood clearance, can potentially limit clinical translation of 99mTc-RP805 as an effective MMP tracer for cardiovascular applications. In addition, the limited hydrosolubility of RP805 precursor is a barrier to establishing uptake specificity in vivo. As such, only a few studies, mainly in vascular and valvular disease models, have attempted to demonstrate uptake specificity of this imaging agent in vivo $(15,17)$. We sought to address these limitations by modifying the structure of the precursor, which would improve hydrophilicity and possibly blood clearance, without affecting MMP binding. As designed, RYM was found to be hydrosoluble, even at relatively high concentrations needed for blocking studies. In addition, in comparison to ${ }^{99 \mathrm{~m}} \mathrm{Tc}-\mathrm{RP} 805$, ${ }^{99 m}$ Tc-RYM1 showed a faster blood clearance, which facilitates early imaging and improves vessel wall-to-blood contrast in vivo. These favorable characteristics were empirically demonstrated in AAA small-animal SPECT imaging studies, performed starting at $1 \mathrm{~h}$ after injection.

The optimal imaging time depends on many factors, and for vascular imaging the vessel wall-to-blood ratio (contrast) is critical. A longer tracer circulation time would increase tissue uptake, providing that the tracer has not reached a plateau or is increasingly retained, for example, through internalization. We have no empiric evidence or theoretic reason (given the biology of MMPs) to believe there is internalization of these tracers. To the contrary, we expect there will be some tissue washout in the presence of the rapid decline in blood levels. Despite a lower blood level, ${ }^{99 m}$ Tc-RYM1 uptake in many organs was significantly higher than ${ }^{99 \mathrm{~m} T c-R P 805}$ (Fig. 2) and the final activity in urine was lower. This latter point might be explained

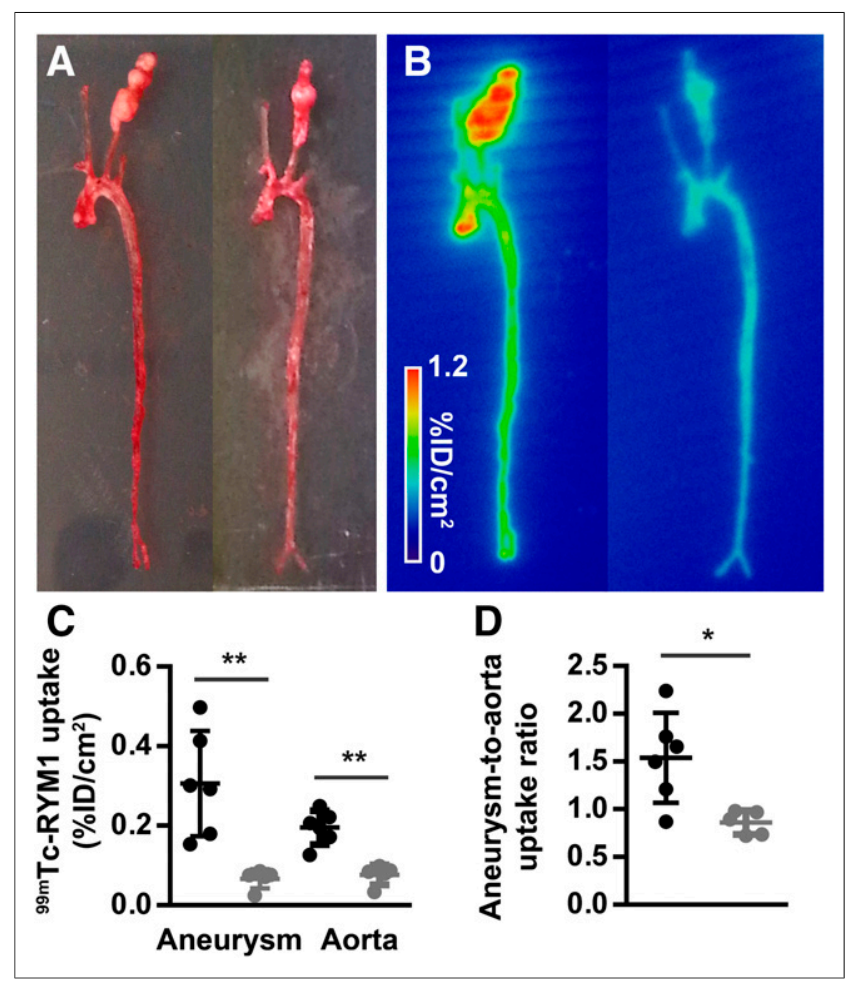

FIGURE 3. 99mTC-RYM1 imaging of carotid aneurysm. (A and B) Examples of ex vivo photography (A) and autoradiography (B) of aortae and carotid arteries from apoE ${ }^{-/-}$mice with $\mathrm{CaCl}_{2}$-induced carotid aneurysm injected with ${ }^{99 m T c-R Y M 1}$ without (left) and with preinjection of excess of MMP inhibitor RYM (right). (C and D) Autoradiographic quan-

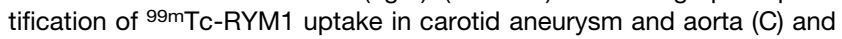
aneurysm-to-aorta uptake ratio (D) for control $(\bullet)$ and blocking $(\bullet)$ groups. ID: injected dose. ${ }^{\star} P<0.05 .{ }^{* \star} P<0.01$. 


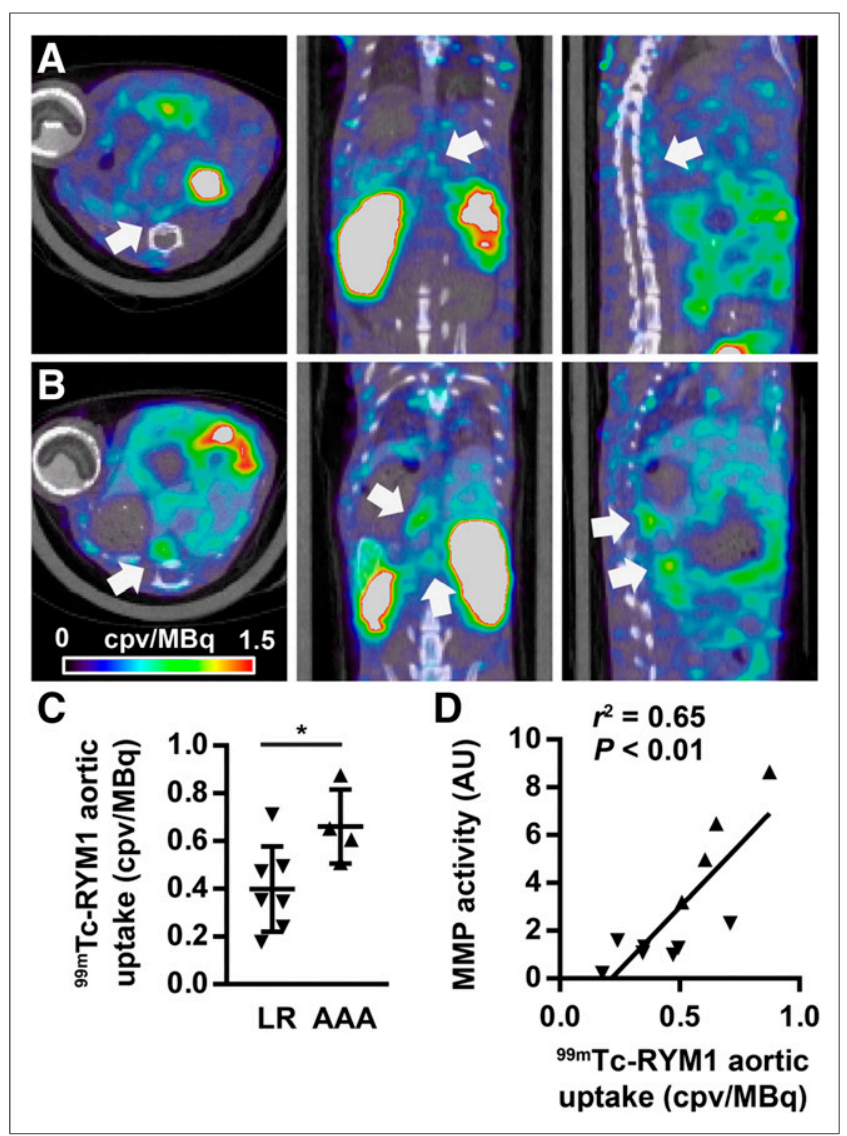

FIGURE 4. ${ }^{99 m T C-R Y M 1}$ imaging of AAA. (A and $B$ ) Examples of fused 99mTc-RYM1 SPECT/CT images of animals from the low remodeling (A) and aneurysm (B) groups, classified on the basis of visual in situ analysis of abdominal aorta. Transversal (left), coronal (middle), and sagittal (right) views are shown. Arrows point to areas of maximal tracer uptake in abdominal aorta. (C) Quantification of ${ }^{99 \mathrm{~m} T c-R Y M 1}$ signal in area of maximal tracer uptake in suprarenal abdominal aorta in low remodeling and AAA groups. ${ }^{*} P<0.05$. (D) Correlation between ${ }^{99 m T C-R Y M 1}$ signal in vivo and MMP activity quantified by zymography ex vivo. $A U=$ arbitrary units; $\mathrm{cpv}=$ counts per voxel; $\mathrm{LR}=$ low remodeling.

by a combination of high first-pass clearance, higher global tissue uptake, and random nature of urine samples. In vivo blocking studies (Supplemental Fig. 3) showed that the major component of tissue uptake is specific, reflecting a hitherto less recognized basal MMP activation in those tissues. This higher uptake, which indicates a higher sensitivity for the target, is not explained by differences in MMP affinity, as both tracers have comparable affinities toward MMPs, and may reflect better tissue penetration and easier accessibility to the target of ${ }^{99 m}$ Tc-RYM1. Importantly, in contrast to many other tissues, there was a trend toward higher uptake of ${ }^{99 m} \mathrm{Tc}-\mathrm{RP} 805$ than ${ }^{99 \mathrm{~m} \mathrm{Tc}-}$ RYM1 in the normal aorta at $2 \mathrm{~h}$ after injection. This basal uptake in the normal vessel is a major disadvantage for ${ }^{99 \mathrm{~m} T c-R P 805}$ and highlights the necessity of blocking studies with the nonlabeled precursor to demonstrate specificity for any imaging application, whether vascular or not. In the absence of such blocking studies, it is impossible to ascertain the validity of the conclusions of any study. As an alternative, other MMP inhibitors may be used in vivo to demonstrate signal specificity. However, caution is warranted in interpreting the data obtained with chronic administration of MMP inhibitors, because a reduction in the signal may reflect changes in disease biology (e.g., inflammation), which reduce nonspecific uptake of the tracers.
Evaluation of ${ }^{99 \mathrm{~m} T c-R Y M 1}$ in 2 preclinical models not only demonstrated the feasibility of in vivo imaging in aneurysm, but also provided complementary information regarding ${ }^{99 m} \mathrm{Tc}-\mathrm{RYM} 1$ uptake in aneurysm. Preinjection of an excess of RYM in the carotid aneurysm model resulted in a significant decrease in ${ }^{99 m} \mathrm{Tc}-\mathrm{RYM} 1$ uptake in aneurysm, establishing uptake specificity of the tracer. Consistent with a certain level of basal MMP activation in the normal artery, this blocking also led to a reduction in aortic tracer uptake. The more prominent blocking effect in aneurysm, reflected in a reduced aneurysm-to-aorta uptake ratio under blocking conditions, mirrored the higher level of MMP activation in aneurysm. In Ang II-infused mice, animals that had developed aneurysm showed higher tracer uptake in suprarenal abdominal aorta. Importantly, there was no correlation between aortic size and MMP signal in vivo, indicating that this enhanced uptake is not primarily related with aortic size. Of note, tracer uptake was often higher in aneurysm shoulders, at the border of areas of arterial enlargement (Supplemental Fig. 4). Although a detailed evaluation of this observation is beyond the scope of this report, it may suggest the presence of active arterial remodeling in these areas.

We leveraged the heterogeneity of the response to Ang II infusion in apoE ${ }^{-1-}$ mice to investigate ${ }^{99 \mathrm{~m}} \mathrm{Tc}-\mathrm{RYM} 1$ uptake and its correlates in AAA. The biologic basis of this heterogeneity is unclear and remains beyond the scope of this study. We cannot exclude the possibility that Ang II infusion increases aortic MMP signal even in animals without evident AAA. In a previous study using the same animal model, the threshold for aortic enlargement was set at a diameter of $0.97 \mathrm{~mm}$ based on aortic size in control, saline-infused animals (5). The mean external aortic diameter in our low remodeling group was slightly above this $0.97-\mathrm{mm}$ threshold. Nevertheless, 99mTc-RYM1 signal was significantly higher in the AAA group and correlated well with aortic MMP activity detected by zymography ex vivo. Tissue MMP activity is tightly regulated at several levels, including MMP gene expression, MMP activation (through proteolytic cleavage of the pro-domain or allosteric activation) (19), and the presence of endogenous inhibitors (e.g., tissue inhibitors of MMPs) (20). We found a significant correlation between in vivo aortic 99mTc-RYM1 signal with MMP-12 messenger RNA expression in Ang II-infused animals. Inflammatory cells are major sources of MMP production, and protease activity is closely linked to tissue inflammation (21). The link between macrophages and MMP-12 (macrophage elastase) is particularly relevant in this regard (22). Accordingly, we found strong correlations between aortic 99mTc-RYM1 signal, MMP activity, and MMP-12 expression on one hand, and macrophage marker CD68 expression on the other hand.

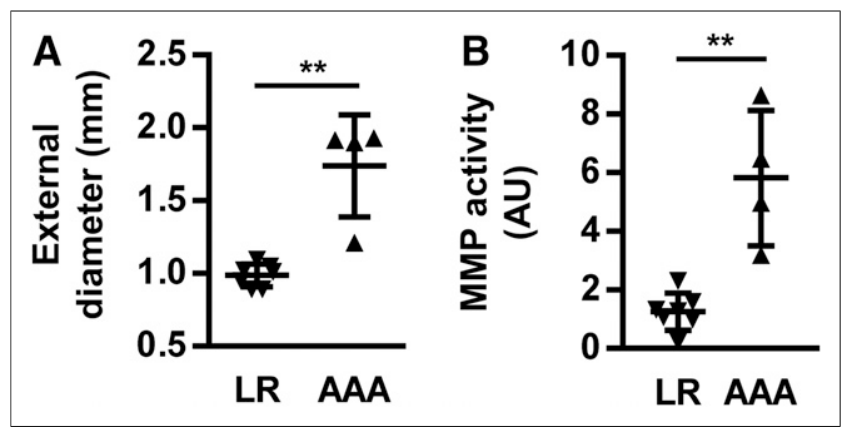

FIGURE 5. Ex vivo characterization of suprarenal abdominal aorta. (A) Maximal external diameter of abdominal aorta in low remodeling and AAA groups. (B) Aortic MMP activity quantified by zymography in low remodeling and AAA groups. ${ }^{\star} P<0.01$. $\mathrm{AU}=$ arbitrary units; $\mathrm{LR}=$ low remodeling. 


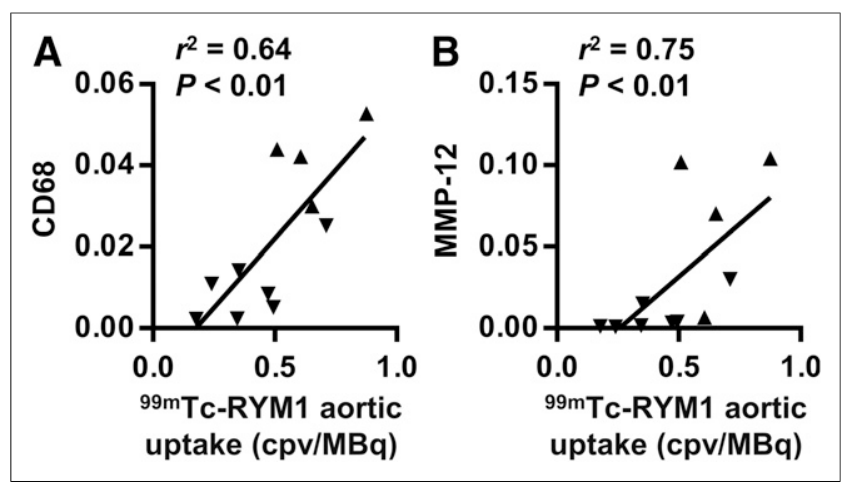

FIGURE 6. Correlates of aortic ${ }^{99 m T C-R Y M 1}$ signal on in vivo smallanimal SPECT/CT images. Correlation between suprarenal abdominal aorta ${ }^{99 m T c-R Y M 1}$ uptake and $\beta$-actin-normalized CD68 (A) and MMP12 (B) gene expression. $\mathrm{cpv}=$ counts per voxel.

A direct comparison of ${ }^{99 m}$ Tc-RYM1 with ${ }^{99 m} \mathrm{Tc}-\mathrm{RP} 805$ is beyond the scope of this study. Qualitatively, whereas both tracers share similar affinity profiles for activated MMPs (11), higher uptake of ${ }^{99 m}$ Tc-RYM1 was observed in various tissues. This is apparent when comparing SPECT images with 99mTc-RYM1 with those obtained with ${ }^{99 \mathrm{~m} T c-R P 805}$ in a similar animal model (5). As shown by the blocking study, a large portion of this uptake is specific, and related to basal MMP activity. However, because not directly in the vicinity of the vessel wall, this increased uptake should not have influenced tracer uptake quantification in AAA, and unlike blood activity is less relevant to imaging in larger models.

In addition to AAA, dysregulated MMP activity is involved in other cardiovascular disorders, such as left-ventricle remodeling postmyocardial infarction (23) and atherosclerosis (24), as well as many primarily noncardiovascular pathologies, including neurodegenerative diseases and cancer. Therefore, ${ }^{99 \mathrm{~m}}$ Tc-RYM1 molecular imaging could improve diagnosis and assessment of therapeutic response and influence patient management in a wide range of applications. In clinical trials, several nonselective MMP inhibitors have shown adverse effects that preclude their use as therapeutic agents $(25,26)$. Although we do not know whether RYM has limitations similar to a therapeutic agent, the tracer dose of RYM1 used for imaging applications is not expected to result in side effects. With regards to imaging targets, selective targeting of specific MMPs is potentially of interest $(22,24,27)$. However, the evaluation of global proteolytic activity of MMPs, more integrative of different processes at play, could prove to be more informative, especially if the signal is stronger and less prone to background noise.

\section{CONCLUSION}

${ }^{99 m}$ Tc-RYM1 is a novel MMP-targeted tracer, with favorable pharmacokinetics for early in vivo imaging. In vivo, ${ }^{99 m}$ Tc-RYM1 signal in aneurysm is specific and correlates with MMP activity and inflammation in murine AAA. If validated in humans, ${ }^{99 \mathrm{~m} T c-R Y M 1-b a s e d}$ molecular imaging may improve patient management in AAA as well as other disorders associated with dysregulated MMP activity.

\section{DISCLOSURE}

This work was supported by grants from NIH (R01-HL112992, R01-HL114703), Connecticut Department of Public Health (20160087), and Department of Veterans Affairs (I0-BX001750). No other potential conflict of interest relevant to this article was reported.

\section{ACKNOWLEDGMENT}

We thank Dr. Mousumi Ghosh at Yale West Campus Analytical Core for her assistance in preparing the tracers.

\section{REFERENCES}

1. Nordon IM, Hinchliffe RJ, Loftus IM, Thompson MM. Pathophysiology and epidemiology of abdominal aortic aneurysms. Nat Rev Cardiol. 2011;8:92-102.

2. Toczek J, Meadows JL, Sadeghi MM. Novel molecular imaging approaches to abdominal aortic aneurysm risk stratification. Circ Cardiovasc Imaging. 2016;9: e003023.

3. Sakalihasan N, Limet R, Defawe OD. Abdominal aortic aneurysm. Lancet. 2005;365:1577-1589.

4. Morris DR, Biros E, Cronin O, Kuivaniemi H, Golledge J. The association of genetic variants of matrix metalloproteinases with abdominal aortic aneurysm: a systematic review and meta-analysis. Heart. 2014;100:295-302.

5. Golestani R, Razavian M, Nie L, et al. Imaging vessel wall biology to predict outcome in abdominal aortic aneurysm. Circ Cardiovasc Imaging. 2014;8:e02471.

6. Ye Y, Toczek J, Razavian M, et al. A new arginine-containing macrocyclic hydroxamate analog for MMP-targeted SPECT imaging: design, synthesis, ${ }^{99 \mathrm{~m}}$ Tc-labeling, and evaluation. $J$ Nucl Med. 2016;57(suppl 2):1140.

7. Golestani R, Razavian M, Ye Y, et al. Matrix metalloproteinase-targeted imaging of lung inflammation and remodeling. J Nucl Med. 2017;58:138-143.

8. Devel L, Rogakos V, David A, et al. Development of selective inhibitors and substrate of matrix metalloproteinase-12. J Biol Chem. 2006;281:11152-11160.

9. Razavian M, Zhang J, Nie L, et al. Molecular imaging of matrix metalloproteinase activation to predict murine aneurysm expansion in vivo. $\mathrm{J} \mathrm{Nucl} \mathrm{Med}$. 2010;51:1107-1115.

10. Daugherty A, Manning MW, Cassis LA. Angiotensin II promotes atherosclerotic lesions and aneurysms in apolipoprotein E-deficient mice. J Clin Invest. 2000; 105:1605-1612.

11. Su H, Spinale FG, Dobrucki LW, et al. Noninvasive targeted imaging of matrix metalloproteinase activation in a murine model of postinfarction remodeling. Circulation. 2005;112:3157-3167.

12. Razavian M, Tavakoli S, Zhang J, et al. Atherosclerosis plaque heterogeneity and response to therapy detected by in vivo molecular imaging of matrix metalloproteinase activation. J Nucl Med. 2011;52:1795-1802.

13. Tavakoli S, Razavian M, Zhang J, et al. Matrix metalloproteinase activation predicts amelioration of remodeling after dietary modification in injured arteries. Arterioscler Thromb Vasc Biol. 2011;31:102-109.

14. Razavian M, Nie L, Challa A, et al. Lipid lowering and imaging protease activation in atherosclerosis. J Nucl Cardiol. 2014;21:319-328.

15. Jung JJ, Razavian M, Challa AA, et al. Multimodality and molecular imaging of matrix metalloproteinase activation in calcific aortic valve disease. $\mathrm{J} \mathrm{Nucl} \mathrm{Med}$. 2015;56:933-938.

16. Jung JJ, Razavian M, Kim HY, et al. Matrix metalloproteinase inhibitor, doxycycline and progression of calcific aortic valve disease in hyperlipidemic mice. Sci Rep. 2016;6:32659.

17. Fujimoto S, Hartung D, Ohshima S, et al. Molecular imaging of matrix metalloproteinase in atherosclerotic lesions: resolution with dietary modification and statin therapy. J Am Coll Cardiol. 2008;52:1847-1857.

18. Schäfers M, Riemann B, Kopka K, et al. Scintigraphic imaging of matrix metalloproteinase activity in the arterial wall in vivo. Circulation. 2004;109:2554-2559.

19. Hadler-Olsen E, Fadnes B, Sylte I, Uhlin-Hansen L, Winberg JO. Regulation of matrix metalloproteinase activity in health and disease. FEBS J. 2011;278:28-45.

20. Visse R, Nagase H. Matrix metalloproteinases and tissue inhibitors of metalloproteinases: structure, function, and biochemistry. Circ Res. 2003;92:827-839.

21. Page-McCaw A, Ewald AJ, Werb Z. Matrix metalloproteinases and the regulation of tissue remodelling. Nat Rev Mol Cell Biol. 2007;8:221-233.

22. Razavian M, Bordenave T, Georgiadis D, et al. Optical imaging of MMP-12 active form in inflammation and aneurysm. Sci Rep. 2016;6:38345.

23. Spinale FG. Myocardial matrix remodeling and the matrix metalloproteinases: influence on cardiac form and function. Physiol Rev. 2007;87:1285-1342.

24. Newby AC. Matrix metalloproteinase inhibition therapy for vascular diseases. Vascul Pharmacol. 2012;56:232-244.

25. Vandenbroucke RE, Libert C. Is there new hope for therapeutic matrix metalloproteinase inhibition? Nat Rev Drug Discov. 2014;13:904-927.

26. Peterson JT. The importance of estimating the therapeutic index in the development of matrix metalloproteinase inhibitors. Cardiovasc Res. 2006;69:677687.

27. Bordenave T, Helle M, Beau F, et al. Synthesis and in vitro and in vivo evaluation of MMP-12 selective optical probes. Bioconjug Chem. 2016;27:2407-2417. 